\title{
RESONANCE SPECTRUM FOR ONE-DIMENSIONAL LAYERED MEDIA
}

\author{
ALEXEI IANTCHENKO
}

\begin{abstract}
We consider the "weighted" operator $P_{k}=-\partial_{x} a(x) \partial_{x}$ on the line with a steplike coefficient which appears when propagation of waves thorough a finite slab of a periodic medium is studied. The medium is transparent at certain resonant frequencies which are related to the complex resonance spectrum of $P_{k}$.

If the coefficient is periodic on a finite interval (locally periodic) with $k$ identical cells then the resonance spectrum of $P_{k}$ has band structure. In the present paper we study a transition to semi-infinite medium by taking the limit $k \rightarrow \infty$. The bands of resonances in the complex lower half plane are localized below the band spectrum of the corresponding periodic problem $(k=\infty)$ with $k-1$ or $k$ resonances in each band. We prove that as $k \rightarrow \infty$ the resonance spectrum converges to the real axis.
\end{abstract}

\section{INTRODUCTION}

In the present paper we consider operator $P_{k}=-\partial_{x} a_{k}(x) \partial_{x}$ on the line with step-like coefficient $a_{k}$ which is periodic on a finite interval defined as follows:

$$
a_{k}(x)=a(x) \text {, for } x \in[0, k] ; a_{k}(x)=\frac{1}{b_{1}^{2}}, \text { for } x \notin[0, k],
$$

where $a(x)$ is 1 -periodic function equal to

$$
a_{0}(x)= \begin{cases}b_{2}^{-2} & \text { for } x \in\left[0, x_{2}\right) \\ b_{1}^{-2} & \text { for } x \in\left[x_{2}, 1\right)\end{cases}
$$

for $x \in[0,1)$. Here $b_{1,2}>0$ and $0<x_{2}<1$. Equation

$$
P_{k} \psi=-\partial_{x} a_{k}(x) \partial_{x} \psi(x)=\lambda^{2} \psi
$$

appears when the propagation of waves through a finite slab of a periodic medium is studied. Such systems are also called finite or locally periodic media (for revue see [6]).

When $k$ is large then the properties of medium is close to an infinite periodic problem in a sense that we are going to discuss in the present paper.

We denote $P=-\partial_{x} a(x) \partial_{x}$ the pure periodic operator, where $a(x)$ is 1 -periodic function equal to $a_{0}$ for $x \in[0,1)$ as in (2). Then the Floquet theory shows the existence of a pair of the quasi-periodic solutions $\psi_{ \pm}$of the equation $-\partial_{x} a(x) \partial_{x} \psi_{ \pm}=\lambda^{2} \psi_{ \pm}$,

$$
\psi^{ \pm}(\lambda, x+1)=e^{ \pm i \theta} \psi^{ \pm}(\lambda, x),
$$

such that $\psi^{ \pm} \in L^{2}\left(\mathbb{R}_{ \pm}\right)$for $\operatorname{Im} \lambda>0$. Here $\theta=\theta(\lambda)$ is the Bloch phase. We denote

$$
F(\lambda)=\frac{\rho+1}{2} \cos \left\{\lambda\left(x_{2} b_{2}+\left(1-x_{2}\right) b_{1}\right)\right\}-\frac{\rho-1}{2} \cos \left\{\lambda\left(x_{2} b_{2}-\left(1-x_{2}\right) b_{1}\right)\right\}
$$

Date: November 20, 2018.

2000 Mathematics Subject Classification. 47A10, 47A40, 81Q10.

Key words and phrases. One-dimensional, layered, truncated periodic, scattering resonances. 
the Lyaponov function for $P$ (see Section 2.1). Here

$$
\rho=\frac{b_{1}^{2}+b_{2}^{2}}{2 b_{1} b_{2}} .
$$

The spectrum of the operator $P$ has band structure with allowed zones defined as follows:

$$
\lambda \in \sigma(P) \Leftrightarrow|F(\lambda)|^{2}<1, \lambda \in \mathbb{R}
$$

(see 4] and Section 4). The band edges are given by solutions of $F(\lambda)= \pm 1$.

The relation between the Bloch phase $\theta$ and the spectral parameter $\lambda$ is called dispersion relation:

$$
\cos \theta(\lambda)=F(\lambda)
$$

Since the coefficient $a(x)$ is constant equal to $1 / b_{1}^{2}$ outside a finite region, we are here concerned with a scattering problem.

We shall denote the reflection and transmission coefficients for the operator $P_{k}$ by $r_{k}$ and $t_{k}$, respectively:

$$
P_{k} \psi=\lambda^{2} \psi, \psi=e^{i \lambda b_{1} x}+r_{k} e^{-i \lambda b_{1} x}, x<0 ; \psi=t_{k} e^{i \lambda b_{1} x}, x>k .
$$

Following the ideas in [7] we consider a transition to semi-infinite periodic materials by taking the limit $k \rightarrow \infty$ of the reflection coefficient $r_{k}$ for $P_{k}$.

The limiting operator

$$
P_{\infty} \psi=-\partial_{x} a_{\infty}(x) \partial_{x} \psi(x)
$$

corresponds to the case of such a long slab that it can be considered as half infinite.

In the case of the operator $P_{\infty}$, the solution $\psi$ of the scattering problem is defined as the solution of the equation $P_{\infty} \psi=\lambda^{2} \psi$, such that

$$
\psi=e^{i \lambda b_{1} x}+r e^{-i \lambda b_{1} x}, x<0 ; \psi=c \psi^{+}(\lambda, x), x>0,
$$

with some $r=r(\lambda), c=c(\lambda)$.

As in 7] we have that the reflection coefficients $r_{k}(\lambda)$ and $r(\lambda)$ are analytic in the upper half plane $\mathbb{C}_{+}=\{\lambda: \operatorname{Im} \lambda>0\}$ and continuous in $\overline{\mathbb{C}}_{+}$, and $r_{k}(\lambda) \rightarrow r(\lambda)$ when $k \rightarrow \infty$ and $\lambda \in \mathbb{C}_{+}$. When $\lambda$ is real, $r_{k}(\lambda)$ converges to $r(\lambda)$ in the weak sense (see Theorem 2 . Section 2.2).

Numerical calculation shows that in each allowed zone of $\sigma(P)$ there are in general $k-1$ frequencies $\lambda_{j}$ where the transmission probability is one: $\left|t_{k}\left(\lambda_{j}\right)\right|^{2}=1, j=1, \ldots, k-1$, and the medium is perfectly transparent: $\left|r_{k}\left(\lambda_{j}\right)\right|^{2}=0$. There exist an additional frequency $\lambda_{0}$ when the medium consisting of only one unit cell is transparent and then $\left|t_{n}\left(\lambda_{0}\right)\right|^{2}=1$ for all $n=1,2, \ldots, k$. The pics in the transmission probability are related to the complex resonances close to the real axis.

We make the following definition.

The operator $P_{k}$ defined from $\left\{u \in H^{1}(\mathbb{R}), a \partial_{x} u \in H^{1}(\mathbb{R})\right\}$ to $L^{2}(\mathbb{R})$ is self-adjoint. For $\operatorname{Im} \lambda>0$, we call $R_{k}(\lambda) v=\left(-\partial_{x} a(x) \partial_{x}-\lambda^{2}\right)^{-1} v$ the resolvent of $P_{k}$. For any $k=1,2, \ldots$, the operator-valued function

$$
R_{k}(\lambda): L_{\text {comp }}^{2}(\mathbb{R}) \mapsto L_{\text {loc }}^{2}(\mathbb{R})
$$

can be continued to the lower complex half-plane $\mathbb{C}_{-}$as a meromorphic function of $\lambda \in \mathbb{C}$ and it has no poles for $\operatorname{Im} \lambda \geq-\epsilon_{k}, \lambda \neq 0$, with $\epsilon_{k}>0$ positive constant dependent on $k$ (see Section (4).

The poles of the $R_{k}(\lambda)$ in $\mathbb{C}_{-}$are called resonances or scattering poles. We denote the set of resonances $\operatorname{Res}\left(P_{k}\right)$.

Using the explicit construction of the resolvent in [4 the poles are calculated numerically. Some examples are presented in Section A, figures (1), (2) and (3). We summarize the properties of $\operatorname{Res}\left(P_{k}\right)$ in the following Theorem. 
Theorem 1. We consider the finite periodic $P_{k}$ and periodic $P$ operators generated by the same unit cell given in (2). Let $\operatorname{Res}\left(P_{k}\right) \subset \mathbb{C}_{-}$denote the resonance spectrum for the finite periodic system with $k=2,3 \ldots$ identical cells and $\sigma(P) \subset \mathbb{R}$ denote the band spectrum for $P$ given by (4).

The resonance spectrum for the finitely periodic system has band structure related to the bands of the real spectrum for the pure periodic problem as follows:

1) The resonance spectrum of $P_{k}$ has band structure. Resonances are localized below the bands of the real spectrum of $P$ :

$$
\lambda \in \operatorname{Res}\left(P_{k}\right) \Rightarrow \operatorname{Re}(\lambda) \text { satisfies (4) } \Leftrightarrow \operatorname{Re}(\lambda) \in \sigma\left(P_{\infty}\right) .
$$

Each resonance band of $P_{k}$ consists of $k-1$ resonances $\lambda_{1}, \ldots, \lambda_{k}-1$ and eventually an additional resonance with real part $\operatorname{Re} \lambda=\lambda_{0}=\pi m / x_{2} b_{2}, m \in \mathbb{Z}$, such that $\left|t_{1}\left(\lambda_{0}\right)\right|^{2}=1$, the one-cell medium is "perfectly transparent" at frequency $\lambda_{0}$.

2) If the condition

$$
b_{2} x_{2}=b_{1}\left(1-x_{2}\right) \Leftrightarrow \frac{b_{1}}{x_{2}}=\frac{b_{2}}{1-x_{2}} .
$$

is satisfied then $\lambda_{0}=\pi m / x_{2} b_{2}, m \in \mathbb{Z}$, is the degenerate band edge (two bands has common edge at $\left.\lambda_{0}\right)$. The resonance spectrum $\operatorname{Res}\left(P_{k}\right), k=1,2,3, \ldots$, is periodic with the period $T=\frac{\pi}{b_{2} x_{2}}$.

3) As $k \rightarrow \infty$ then the resonance spectrum of $P_{k}$ approaches the real axis.

In the present paper we motivate these numerical results.

The band structure of the resonance spectrum for a finitely periodic system and its relation to the band spectrum of the correspondent periodic problem is well-known in physical literature (see [1]).

We say that $\operatorname{Res}\left(P_{k}\right)$ is periodic if there exists $T>0$, period, such that

$$
\operatorname{Res}\left(P_{k}\right) \cap([q+T n, p+T n]-i \mathbb{R})=\operatorname{Res}\left(P_{k}\right) \cap([q+T m, p+T m]-i \mathbb{R})
$$

for any $q<p$ and $n, m \in \mathbb{Z}$. This property follows directly from the equations defining the resonances in Section (4.2) if condition (6) is satisfied.

A special property of the operator $P=-\partial_{x} a(x) \partial_{x}$ with step-like periodic coefficient $a(x)$ is that the coefficients $(r \pm 1)$ in the dispersion relation

$$
2 \cos \theta(\lambda)=(\rho+1) \cos \left\{\lambda\left(x_{2} b_{2}+\left(1-x_{2}\right) b_{1}\right)\right\}-(\rho-1) \cos \left\{\lambda\left(x_{2} b_{2}-\left(1-x_{2}\right) b_{1}\right)\right\}
$$

are independent of the spectral parameter $\lambda$. Formula (7) implies that the band spectrum is periodic if the profile of $a$ verifies ([6).

The third part of the Theorem is proved in Section 6 .

The convergence of the resonances for a finitely periodic system with $k$ cells to the bands of real spectrum for the periodic problem as $k \rightarrow \infty$ was discussed by F. Barra and P. Gaspard in [3] in the case of Schrödinger equation.

In our proof we use representations for the reflection and transmission coefficients $r_{k}, t_{k}$ for a finite slab of periodic medium as in the recent paper of Molchanov and Vainberg [7]. The authors considered transition of truncated medium described by the 1-D Schrödinger operator to semi-infinite periodic materials. By relating the reflection coefficients to the resolvent of $P_{k}$ we show explicitly that the resonances correspond to the poles of the analytic continuation of $r_{k}(\lambda)$ to $\mathbb{C}_{-}$. Then we consider the limit of the poles of $r_{k}(\lambda)$, as $k \rightarrow \infty$.

Note that for $\lambda \in \mathbb{R}$ the reflection coefficient $r_{k+1}$ for $k+1$ cells medium is related to $r_{k}$ for $k$ cells medium via $r_{k+1}=f_{\lambda}\left(r_{k}\right)$, where $f_{\lambda}$ is a linear-fractional automorphism of the unit disk. By considering the fixed point of $f_{\lambda}$ we get a new proof of the convergence of $r_{k}(\lambda)$ to $r(\lambda)$ when 
$\lambda$ belongs to the spectral gapes and non-degenerate band edges for the operator $P$ (see Section 7).

The structure of the paper is the following:

In Section 2 we recall some well-known facts concerning spectral problem for weighted SturmLiouville operators (see [2]) and consider scattering by a finite slab of a periodic medium. We follow [7] with minor changes due to the special form of operator $P_{k}$. We recall exact formulas for the the reflection and transmission coefficients using the iteration of the monodromy matrix. We recall also the result of [7] on a transition to semi-infinite periodic material (limit $k \rightarrow \infty$ ). In Section 3 we give explicit expression for the monodromy matrix of $P_{k}$. In Section 4 we recall the iterative procedure used in [4] for construction of the resolvent $R_{k}$ and define resonances. In Section 5 the reflection coefficient $r_{k}$ is expressed using the iteration formulas of $[4$ and we show that the poles of $R_{k}(\lambda)$ and the poles of $r_{k}(\lambda), \lambda \in \mathbb{C}_{-}$, coincide. In Section 6 we prove the convergence of Res $\left(P_{k}\right)$ to the real axis. In Section 7 we discuss the convergence of $r_{k}(\lambda)$, $k \rightarrow \infty$, for $\lambda \in \mathbb{R}$ by considering the limit of a sequence of linear-fractional automorphisms on the unit disk. In Appendix $\AA$ we present numerical examples.

AcKnowledgements. The author would like to thank Maciej Zworski for suggesting to look at the problem considered in the present paper and for helpful discussions.

\section{General methods For trunCAted PeRiodic operators}

In this section we following [7] consider the scattering theory for operator $P_{k}$ combining the Floquet-Bloch theory and scattering theory for $1-\mathrm{D}$ weighted operators.

2.1. The monodromy matrix and Bloch quasi-momentum. We recall first some wellknown facts concerning the spectral problem of Sturm-Liouville operators on the line (see [2]). We consider equation

$$
P \psi=-\partial_{x} a(x) \partial_{x} \psi(x)=\lambda^{2} \psi
$$

on $\left\{\psi \in H_{\mathrm{loc}}^{1}(\mathbb{R}), a \partial_{x} \psi \in H_{\mathrm{loc}}^{1}(\mathbb{R})\right\}$ with a strictly positive $a(x)$ as in the Introduction, formula (11) or periodic as $a_{0}$ in (2).

Let $\psi_{1,2}$ be solutions of (8) with initial data

$$
\psi_{1}(\lambda, 0)=1,\left(a \partial_{x} \psi_{1}\right)(\lambda, 0)=0 ; \psi_{2}(\lambda, 0)=0,\left(a \partial_{x} \psi_{2}\right)(\lambda, 0)=1 .
$$

We define the transfer matrix (propagator) $M_{\lambda}(0, x)$ for operator $P$

$$
M_{\lambda}(0, x)=\left(\begin{array}{cc}
\psi_{1}(\lambda, x) & \lambda \psi_{2}(\lambda, x) \\
\frac{\left(a \partial_{x} \psi_{1}\right)(\lambda, x)}{\lambda} & \left(a \partial_{x} \psi_{2}\right)(\lambda, x)
\end{array}\right) .
$$

From (9) it follows that $M_{\lambda}(0, x)$ is the identity matrix. For any solution $\psi$ of (8) matrix $M_{\lambda}(0, x)$ maps the Cauchy data of $\psi$ at $x=0$ into the Cauchy data of $\psi$ at point $x$ :

$$
M_{\lambda}(0, x):\left(\begin{array}{c}
\psi(0) \\
\frac{\left(a \partial_{x} \psi\right)(\lambda, 0)}{\lambda}
\end{array}\right) \mapsto\left(\begin{array}{c}
\psi(x) \\
\frac{\left(a \partial_{x} \psi\right)(\lambda, x)}{\lambda}
\end{array}\right) .
$$

As the generalized Wronskian associated with $\psi_{1}, \psi_{2}$

$$
W\left[\psi_{1}, \psi_{2}\right]=\psi_{1} a \partial_{x} \psi_{2}-\psi_{2} a \partial_{x} \psi_{1}
$$

is constant, we have

$$
\operatorname{det} M_{\lambda}(0, x)=W\left[\psi_{1}, \psi_{2}\right](1)=W\left[\psi_{1}, \psi_{2}\right](0)=1 .
$$

Equation (8) with $\operatorname{Im} \lambda>0$ has exactly one solution $\psi^{+}$in $L^{2}\left(\mathbb{R}_{+}\right)$normalized by the condition $\psi^{+}(\lambda, 0)=1$, and it has exactly one solution $\psi^{-} \in L^{2}\left(\mathbb{R}_{-}\right)$normalized by the same condition. 
Here $\mathbb{R}_{ \pm}$are semiaxes $x \gtrless 0$. Any solution of (8) can be represented as linear combinations of $\psi_{1}$ and $\psi_{2}$ and from the normalization of $\psi^{ \pm}$it follows, that there exist functions $m^{ \pm}=m^{ \pm}(\lambda)$ such that

$$
\psi^{ \pm}=\psi_{1}+m^{ \pm}(\lambda) \psi_{2}, \operatorname{Im} \lambda>0 .
$$

Functions $m^{ \pm}=m^{ \pm}(\lambda)$ are called Weyl's functions and we have

$$
\psi_{1}+m^{+}(\lambda) \psi_{2} \in L^{2}\left(\mathbb{R}_{+}\right), \psi_{1}+m^{-}(\lambda) \psi_{2} \in L^{2}\left(\mathbb{R}_{-}\right), \operatorname{Im} \lambda>0 .
$$

Let $a(x)$ be periodic: $a(x+l)=a(x)$. Consider propagator through one period (monodromy matrix):

$$
M_{\lambda}=M_{\lambda}(0, l)=\left(\begin{array}{cc}
\alpha & \beta \\
\gamma & \delta
\end{array}\right)(\lambda)=\left(\begin{array}{cc}
\psi_{1}(\lambda, l) & \lambda \psi_{2}(\lambda, l) \\
\frac{\left(a \partial_{x} \psi_{1}\right)(\lambda, l)}{\lambda} & \left(a \partial_{x} \psi_{2}\right)(\lambda, l)
\end{array}\right) .
$$

Denote $F(\lambda)=\frac{1}{2} \operatorname{tr} M_{\lambda}=(\alpha+\delta) / 2=\left(\psi_{1}(\lambda, 1)+a \partial \psi_{2}(\lambda, 1)\right.$ the Lyapunov function. Both $M_{\lambda}$ and $F(\lambda)$ are entire function of $\lambda$ and $\operatorname{det} M_{\lambda}=1$. The eigenvalues $\mu^{ \pm}(\lambda)$ of $M_{\lambda}$ are the roots of the characteristic equation

$$
\mu^{2}-2 \mu F(\lambda)+1=0
$$

If $\operatorname{Im} \lambda>0$ then one can select roots $\mu^{ \pm}(\lambda)$ of (11) in such a way that $\mu^{ \pm}(\lambda)=e^{ \pm i l \theta(\lambda)}$, where $\theta(\lambda)$ is analytic and

$$
\operatorname{Im} \theta(\lambda)>0 \text { when } \operatorname{Im} \lambda>0
$$

$$
\left|\mu^{+}(\lambda)\right|<1,\left|\mu^{-}(\lambda)\right|>1, \operatorname{Im} \lambda>0
$$

The roots $\mu^{ \pm}(\lambda)$ for real $\lambda \geq 0$ are defined by continuity in the upper half plane:

$$
\mu^{ \pm}(\lambda)=\mu^{ \pm}(\lambda+i 0), \lambda \in[0, \infty)
$$

Since the trace of $M_{\lambda}$ is equal to the sum of the eigenvalues $e^{ \pm i l \theta(\lambda)}$,

$$
\cos l \theta=F(\lambda)=\frac{1}{2}\left(\psi_{1}+a \psi_{2}^{\prime}\right)(\lambda, l)=\frac{1}{2}(\alpha+\delta) .
$$

The spectrum of $P$ belongs to the positive part of the energy axis $E=\lambda^{2}$ and has band structure.

For real $\lambda \geq 0$, the inequality $|F(\lambda)| \leq 1$ defines the spectral bands (zones)

$$
b_{n}=\left[\lambda_{2 n-1}, \lambda_{2 n}\right], n=1,2, \ldots,
$$

on the frequency axis $\lambda=\sqrt{E}$. The bands are defined by the condition $|F(\lambda)| \leq 1$ and $F(\lambda)= \pm 1$ at any band edge $\lambda=\lambda_{j}$.

The function $\theta(\lambda)$ is real valued when $\lambda$ belongs to a band. The roots $\mu^{ \pm}(\lambda)$ are complex adjoint there, and $\left|\mu^{ \pm}\right|=1$.

The spectrum of $P\left(\right.$ on $\left.L^{2}(\mathbb{R})\right)$ on the frequency axis is $\bigcup_{n=1}^{\infty} b_{n}$.

The complimentary open set, given by $|F(\lambda)|>1$, corresponds to spectral gaps, $\bigcup_{n=1}^{\infty} g_{n}$. On gaps, the function $i \theta(\lambda)$ is real valued, the roots $\mu^{ \pm}(\lambda)$ are real and (13) holds.

A point $\lambda_{j}$ which belongs to the boundary of a band and the boundary of a gap is called a non-degenerate band edge. If it belongs to the boundary of two different bands, it is called a degenerate band edge.

As in [7] we get that if $\lambda=\lambda_{0}$ is a non-degenerate band edge, then $F^{\prime}(\lambda) \neq 0$. If $\lambda=\lambda_{0}$ is a degenerate edge, then $F^{\prime}(\lambda)=0, F^{\prime \prime}(\lambda) \neq 0$. Both eigenvalues of the monodromy matrix $M_{\lambda}$ at any band edge are equal to 1 or both are equal to -1 . 
We normalize the eigenvectors $h^{ \pm}(\lambda)$ of $M_{\lambda}$ by choosing the first coordinate of $h^{ \pm}(\lambda)$ to be equal to one:

$$
h^{ \pm}(\lambda)=\left(\begin{array}{c}
1 \\
m^{ \pm}(\lambda)
\end{array}\right) .
$$

The second coordinates of the vectors $h^{ \pm}(\lambda)$ coincide with the Weyl's functions defined above. In fact, if $\psi^{ \pm}$are solutions of the equation $P \psi=\lambda^{2} \psi$ with the initial Cauchy data given by the eigenvector $h^{ \pm}$, then

$$
\psi^{ \pm}(\lambda, x+l)=e^{ \pm i l \theta(\lambda)} \psi^{ \pm}(\lambda, x)
$$

and (13) implies that $\psi^{ \pm} \in L^{2}\left(\mathbb{R}_{ \pm}\right)$when $\operatorname{Im} \lambda>0$. From here it follows that $\psi^{ \pm}$coincide with Weyl's solution introduced for general Hamiltonians $P$, and that the second coordinates of the vectors $h^{ \pm}(\lambda)$ are Weyl's functions.

Since

$$
\left(\begin{array}{cc}
\alpha-e^{ \pm i l \theta(\lambda)} & \beta \\
\gamma & \delta-e^{ \pm i l \theta(\lambda)}
\end{array}\right)\left(\begin{array}{c}
1 \\
m^{ \pm}
\end{array}\right)=0,
$$

the following two representations are valid for Weyl's functions:

$$
m^{ \pm}(\lambda)=\frac{e^{ \pm i l \theta(\lambda)}-\alpha(\lambda)}{\beta(\lambda)}=\frac{\gamma(\lambda)}{e^{ \pm i l \theta(\lambda)}-\delta(\lambda)} .
$$

2.2. Reflection coefficient for the truncated periodic operator. We consider operator $P_{k}$ with the truncated periodic coefficient $a_{k}$ :

$$
P_{k} \psi=-\partial_{x} a_{k}(x) \partial_{x} \psi(x), a_{k}(x)=a(x), \text { for } x \in[0, k l] ; a_{k}(x)=\frac{1}{b_{1}^{2}}, \text { for } x \notin[0, k l],
$$

which appears when the propagation of waves through a finite slab of a periodic medium is studied. We shall also consider the limiting case $k=\infty$ :

$$
P_{\infty} \psi=-\partial_{x} a_{\infty}(x) \partial_{x} \psi(x),
$$

which corresponds to the case of such a long slab that it can be considered as half infinite.

We shall denote the reflection and transmission coefficients for the operator $P_{k}$ (with compactly supported coefficient $a_{k}$ ) by $r_{k}$ and $t_{k}$, respectively:

$$
P_{k} \psi=\lambda^{2} \psi, \psi=e^{i \lambda b_{1} x}+r_{k} e^{-i \lambda b_{1} x}, x<0 ; \psi=t_{k} e^{i \lambda b_{1} x}, x>k l .
$$

In the case of the operator $P_{\infty}$, the solution $\psi$ of the scattering problem is defined as the solution of the equation $P_{\infty} \psi=\lambda^{2} \psi$, such that

$$
\psi=e^{i \lambda b_{1} x}+r e^{-i \lambda b_{1} x}, x<0 ; \psi=c \psi^{+}(\lambda, x), x>0,
$$

with some $r=r(\lambda), c=c(\lambda)$. We have the following version of Theorem 3 of S. Molchanov, B. Vainberg in [7]:

Theorem 2. 1) The transfer matrix over $k$ periods $M_{\lambda}^{k}=T_{\lambda}(0, l k)$ has the form

$$
M_{\lambda}^{k}=\left(\begin{array}{cc}
\alpha_{k} & \beta_{k} \\
\gamma_{k} & \delta_{k}
\end{array}\right)=\frac{\sin k l \theta(\lambda)}{\sin l \theta(\lambda)} M_{\lambda}-\frac{\sin (k-1) l \theta(\lambda)}{\sin l \theta(\lambda)} I,
$$

where $\theta=\theta(\lambda)$ is the Bloch function. The elements of $M_{\lambda}^{k}$ satisfy the relations

$$
\begin{aligned}
& \alpha_{k}-\delta_{k}=\frac{\sin k l \theta(\lambda)}{\sin l \theta(\lambda)}(\alpha-\delta), \quad \beta_{N}=\frac{\sin k l \theta(\lambda)}{\sin l \theta(\lambda)} \beta, \\
& \gamma_{k}=\frac{\sin k l \theta(\lambda)}{\sin l \theta(\lambda)} \gamma, \quad \alpha_{k}+\delta_{k}=2 \cos k l \theta(\lambda) .
\end{aligned}
$$


2) The reflection coefficients have the forms

$$
\begin{gathered}
r_{k}(\lambda)=-\frac{(\alpha-\delta)+i\left(b_{1} \gamma+\frac{\beta}{b_{1}}\right)}{2 \sin l \theta(\lambda) \frac{\cos k l \theta(\lambda)}{\sin k l \theta(\lambda)}+i\left(b_{1} \gamma-\frac{\beta}{b_{1}}\right)}, \\
r(\lambda)=\frac{\frac{\beta}{b_{1}}+b_{1} \gamma-i(\alpha-\delta)}{2 \sin l \theta(\lambda)-\left(b_{1} \gamma-\frac{\beta}{b_{1}}\right)} .
\end{gathered}
$$

3) The transmission probability have the form

$$
\left|t_{k}(\lambda)\right|^{2}=\frac{4}{\frac{\sin ^{2} l k \theta}{\sin ^{2} l \theta}\left((\alpha-\delta)^{2}+\left(b_{1} \gamma+\frac{\beta}{b_{1}}\right)^{2}\right)+4}=\frac{1}{\frac{\sin ^{2} l k \theta}{\sin ^{2} l \theta} \frac{\left|r_{1}\right|^{2}}{\left|t_{1}\right|^{2}}+1} .
$$

4) The reflection coefficients $r_{k}(\lambda)$ and $r(\lambda)$ are analytic in the upper half plane $\mathbb{C}_{+}=\{\lambda: \operatorname{Im} \lambda>$ $0\}$ and continuous in $\overline{\mathbb{C}}_{+}$. For any $\lambda \in \overline{\mathbb{C}}_{+} \backslash \cup_{n=1}^{\infty} b_{n}$ we have $r_{k}(\lambda) \rightarrow r(\lambda)$. When $\lambda \in \cup_{n=1}^{\infty} b_{n}$, $r_{k}(\lambda)$ converges to $r(\lambda)$ in the weak sense:

$$
\int_{-\infty}^{\infty} r_{k}(\lambda) \varphi(\lambda) d \lambda \rightarrow \int_{-\infty}^{\infty} r(\lambda) \varphi(\lambda) d \lambda \text { as } k \rightarrow \infty .
$$

for any test function $\varphi \in D$.

Proof: We reproduce here the proof of [7] for the sake of completeness with only minor changes due to the "weight" in the definitions of $P_{k}$ and $P_{\infty}$. Formula (18) follows by induction from relation (11):

$$
M_{\lambda}^{2}-2 \cos l \theta(\lambda) M_{\lambda}+I=0 .
$$

The first three relations of (19) are immediate consequences of (18). In order to get the fourth one we note that the eigenvalues of $M_{\lambda}^{k}$ are $\mu^{ \pm}(\lambda)=e^{ \pm i k l \theta(\lambda)}$. Thus,

$$
\alpha_{k}+\delta_{k}=\operatorname{tr} M_{\lambda}^{k}=e^{i k l \theta(\lambda)}+e^{-i k l \theta(\lambda)}=2 \cos k l \theta(\lambda) .
$$

Next we prove (20). The relation between Cauchy data for the left-to-right scattering solution at $x=0$ and $x=k l$ are given by

$$
\left(\begin{array}{cc}
\alpha_{k} & \beta_{k} \\
\gamma_{k} & \delta_{k}
\end{array}\right)\left(\begin{array}{c}
1+r_{k} \\
\frac{i}{b_{1}}\left(1-r_{k}\right)
\end{array}\right)=\left(\begin{array}{c}
t_{k} \\
\frac{i t_{k}}{b_{1}}
\end{array}\right) \Leftrightarrow\left\{\begin{array}{c}
\alpha_{k}\left(1+r_{k}\right)+\beta_{k}\left(\frac{i}{b_{1}}\left(1-r_{k}\right)\right)=t_{k} \\
\gamma_{k}\left(1+r_{k}\right)+\delta_{k}\left(\frac{i}{b_{1}}\left(1-r_{k}\right)\right)=\frac{i t_{k}}{b_{1}}
\end{array}\right.
$$

By dividing the second equation by the first one we arrive at

$$
\frac{\gamma_{k}\left(1+r_{k}\right)+\frac{\delta_{k} i}{b_{1}}\left(1-r_{k}\right)}{\alpha_{k}\left(1+r_{k}\right)+\frac{\beta_{k} i}{b_{1}}\left(1-r_{k}\right)}=\frac{i}{b_{1}} .
$$

Solving for $r_{k}$ we obtain

$$
r_{k}=\frac{\delta_{k}-\alpha_{k}-i\left(b_{1} \gamma_{k}+\frac{\beta_{k}}{b_{1}}\right)}{\delta_{k}+\alpha_{k}+i\left(b_{1} \gamma_{k}-\frac{\beta_{k}}{b_{1}}\right)} .
$$

Using (19) we get

$$
r_{k}=-\frac{\frac{\sin k l \theta}{\sin l \theta}(\alpha-\delta)+i\left(b_{1} \frac{\sin k l \theta}{\sin l \theta} \gamma+\frac{1}{b_{1}} \frac{\sin k l \theta}{\sin l \theta} \beta\right)}{2 \cos k l \theta+i\left(b_{1} \frac{\sin k l \theta}{\sin l \theta} \gamma-\frac{1}{b_{1}} \frac{\sin k l \theta}{\sin l \theta} \beta\right)} .
$$

This justifies (20). 
In order to get (21) we note that (17) implies that

$$
\left(\begin{array}{c}
1+r \\
\frac{i}{b_{1}}(1-r)
\end{array}\right)=c\left(\begin{array}{c}
1 \\
m^{+}
\end{array}\right) \Leftrightarrow \frac{1+r}{\frac{i}{b_{1}}(1-r)}=\frac{1}{m^{+}}
$$

and therefore,

$$
r=\frac{\frac{i}{b_{1}}-m^{+}}{\frac{i}{b_{1}}+m^{+}}
$$

From here and (16) it follows that

$$
r=\frac{\frac{i}{b_{1}}-\frac{e^{i l \theta}-\alpha}{\beta}}{\frac{i}{b_{1}}+\frac{e^{i l \theta}-\alpha}{\beta}}=\frac{\alpha+\frac{i}{b_{1}} \beta-e^{i l \theta}}{e^{i l \theta}-\left(\alpha-\frac{i}{b_{1}} \beta\right)} .
$$

and

$$
r=\frac{\frac{i}{b_{1}}-\frac{\gamma}{e^{i l \theta}-\delta}}{\frac{i}{b_{1}}+\frac{\gamma}{e^{i l \theta}-\delta}}=\frac{\frac{i}{b_{1}}\left(e^{i l \theta}-\delta\right)-\gamma}{\frac{i}{b_{1}}\left(e^{i l \theta}-\delta\right)+\gamma}=\frac{e^{i l \theta}-\left(\delta-i b_{1} \gamma\right)}{e^{i l \theta}-\left(\delta+i b_{1} \gamma\right)} .
$$

Hence,

$$
r\left(2 e^{i l \theta}-\left(\alpha-\frac{i}{b_{1}} \beta\right)-\left(\delta+i b_{1} \gamma\right)\right)=\alpha+\frac{i}{b_{1}} \beta-\left(\delta-i b_{1} \gamma\right)
$$

and

$$
\begin{aligned}
r & =\frac{\left(\alpha+\frac{i}{b_{1}} \beta\right)-\left(\delta-i b_{1} \gamma\right)}{2 e^{i l \theta}-\left(\alpha-\frac{i}{b_{1}} \beta\right)-\left(\delta+i b_{1} \gamma\right)}=\frac{(\alpha-\delta)+i\left(\frac{\beta}{b_{1}}+b_{1} \gamma\right)}{2 e^{i l \theta}-(\alpha+\delta)+i\left(\frac{\beta}{b_{1}}-b_{1} \gamma\right)}= \\
& =\frac{(\alpha-\delta)+i\left(\frac{\beta}{b_{1}}+b_{1} \gamma\right)}{2 i \sin l \theta+i\left(\frac{\beta}{b_{1}}-b_{1} \gamma\right)},
\end{aligned}
$$

where the last equality is a consequence of (14) and it implies (21).

We prove the third statement of the theorem. From (24) it follows

$$
\left|r_{k}\right|^{2}=\frac{\left(\delta_{k}-\alpha_{k}\right)^{2}+\left(b_{1} \gamma_{k}+\frac{\beta_{k}}{b_{1}}\right)^{2}}{\left(\delta_{k}+\alpha_{k}\right)^{2}+\left(b_{1} \gamma_{k}-\frac{\beta_{k}}{b_{1}}\right)^{2}} .
$$

Using that $\left|r_{k}\right|^{2}+\left|t_{k}\right|^{2}=1$ we get

$$
\left|t_{k}\right|^{2}=1-\left|r_{k}\right|^{2}=\frac{4 \delta_{k} \alpha_{k}-4 \gamma_{k} \beta_{k}}{\left(\delta_{k}+\alpha_{k}\right)^{2}+\left(b_{1} \gamma_{k}-\frac{\beta_{k}}{b_{1}}\right)^{2}} .
$$

We use $\operatorname{det} M_{\lambda}^{k}=\alpha_{k} \delta_{k}-\beta_{k} \gamma_{k}=1$ and get

$$
\left|t_{k}\right|^{2}=\frac{4}{4+\left(\delta_{k}-\alpha_{k}\right)^{2}+\left(b_{1} \gamma_{k}+\frac{\beta_{k}}{b_{1}}\right)^{2}}=\frac{4}{\frac{\sin ^{2} l k \theta}{\sin ^{2} l \theta}\left((\alpha-\delta)^{2}+\left(b_{1} \gamma+\frac{\beta}{b_{1}}\right)^{2}\right)+4} .
$$

From formulas (25) and (26) we get

$$
\frac{\left|r_{k}\right|^{2}}{\left|t_{k}\right|^{2}}=\frac{1}{4}\left(\left(\delta_{k}-\alpha_{k}\right)^{2}+\left(b_{1} \gamma_{k}+\frac{\beta_{k}}{b_{1}}\right)^{2}\right)
$$

and hence, putting $k=1$,

$$
\left|t_{k}\right|^{2}=\frac{1}{\frac{\sin ^{2} l k \theta}{\sin ^{2} l \theta} \frac{\left|r_{1}\right|^{2}}{\left|t_{1}\right|^{2}}+1}
$$


The analyticity of $r_{k}(\lambda)$ and $r(\lambda)$ in $\mathbb{C}_{+}$and their continuity in $\overline{\mathbb{C}}_{+}$follow from the explicit formulas (20), (21). For $\lambda \in \mathbb{C}_{+} \bigcup \cup_{n=1}^{\infty} g_{n}$, we have $\operatorname{Im} \theta(\lambda)>0$ and $\theta(\lambda)$ is pure imaginary on the gaps $g_{n}$. Furthermore, if $\operatorname{Im} \theta(\lambda)>0$, then

$$
\frac{\cos k \theta(\lambda)}{\sin k \theta(\lambda)} \rightarrow-i \text { as } k \rightarrow \infty
$$

and this justifies the convergence of $r_{k}(\lambda)$ to $r$ when $\lambda \in \mathbb{C}_{+} \cup \cup_{n=1}^{\infty} g_{n}$.

The weak convergence for $\lambda \in \cup_{n=1}^{\infty} b_{n}$ is a consequence the convergence in the complex half plane.

The proof of Theorem 2 is complete.

Note also the following relations:

$$
\begin{gathered}
\frac{\left|r_{k}\right|^{2}}{\left|t_{k}\right|^{2}}=\frac{\sin ^{2} l k \theta}{\sin ^{2} l \theta} \cdot \frac{\left|r_{1}\right|^{2}}{\left|t_{1}\right|^{2}}, \\
\left|t_{k}\right|^{2}=\frac{4}{4 \cos ^{2} k l \theta+\left(b_{1} \frac{\sin k l \theta}{\sin l \theta} \gamma-\frac{1}{b_{1}} \frac{\sin k l \theta}{\sin l \theta} \beta\right)^{2}}=\frac{4 \frac{\sin ^{2} l \theta}{\sin ^{2} k l \theta}}{4 \sin ^{2} l \theta \frac{\cos ^{2} k l \theta}{\sin ^{2} k l \theta}+\left(b_{1} \gamma-\frac{\beta}{b_{1}}\right)^{2}} .
\end{gathered}
$$

The last formula follows from (26) by using (23).

Formula (22) implies that the perfect transmission $\left(\left|t_{k}\right|^{2}=1\right)$ occurs whenever $\left|r_{1}\right|^{2}=0$ $\left(\left|t_{1}\right|^{2}=1\right)$ or if

$$
\frac{\sin ^{2} l k \theta}{\sin ^{2} l \theta}=0
$$

For $\theta \in[0, \pi / l]$ equation (27) is satisfied when $\theta l k=m \pi$ for $m=1,2, \ldots, k-1$.

Therefore, in the general case $\left(\left|r_{1}\right|^{2} \neq 0\right)$, the transmission probability has $k-1$ peaks with $\left|t_{k}\right|^{2}=1$ in each allowed energy band as $\theta$ increases by $\pi / l$. Since the peaks in the transmission probability (or in general in the cross section) are associated with resonances, we expect to find $k-1$ resonances near each allowed energy band.

On the gaps, the function $i \theta(\lambda)$ is real valued. Then the transmission probability is given by

$$
\left|t_{k}(\lambda)\right|^{2}=\frac{1}{\frac{\sinh ^{2} l k i \theta}{\sinh ^{2} l i \theta} \frac{\left|r_{1}\right|^{2}}{\left|t_{1}\right|^{2}}+1} .
$$

As $\sinh ^{2} l k i \theta \neq 0$ for $\theta \neq 0$, then in the forbidden zone $\left|t_{k}(\lambda)\right|^{2} \neq 1$ unless $\left|t_{1}\right|^{2}=1,\left|r_{1}\right|^{2}=0$. Hence there are no resonances below the gaps.

On the gaps, the reflection coefficient for the half-periodic system $r(\lambda)$ satisfy

$$
|r(\lambda)|^{2}=\frac{\left(\frac{\beta}{b_{1}}+b_{1} \gamma\right)^{2}+(\alpha-\delta)^{2}}{e^{2 i \theta}+e^{-2 i \theta}-2+\left(b_{1} \gamma-\frac{\beta}{b_{1}}\right)^{2}}=\frac{\left(\frac{\beta}{b_{1}}+b_{1} \gamma\right)^{2}+(\alpha-\delta)^{2}}{(\alpha+\delta)^{2}-4+\left(b_{1} \gamma-\frac{\beta}{b_{1}}\right)^{2}}=1
$$

In [7], Theorem 5, was shown that

Lemma 1. If $\lambda_{0}$ is a degenerate band edge, i.e. $F\left(\lambda_{0}\right)= \pm 1, F^{\prime}\left(\lambda_{0}\right)=0$, then the reflection coefficient $r_{k}$ is zero, $r_{k}\left(\lambda_{0}\right)=0$.

The proof uses the fact that at any degenerate band edge $\lambda=\lambda_{0}$ the monodromy matrix $M_{\lambda_{0}}= \pm I$ and $F^{\prime \prime}\left(\lambda_{0}\right) \neq 0$. This allows to pass to the limit in (20) as $\lambda \rightarrow \lambda_{0}$. The numerator in the right hand side of (20) vanishes as $\lambda \rightarrow \lambda_{0}$. The denominator converges to $\pm 2 / k$, since $\theta\left(\lambda_{0}\right)=n \pi$. 
Thus the medium is transparent for the plane wave with the frequency $\lambda_{0}$ and we expect to find a resonance $\lambda \in \mathbb{C}_{-}$below the degenerated band edge $\lambda_{0}$.

\section{The Monodromy MATrix For $P_{k}$}

In this section we give expressions for the elements of the monodromy matrix $M_{\lambda}$.

Let $a(x)$ be 1 -periodic function equal to $a_{0}$ for $x \in[0,1)$ as in (11), (2):

$$
a_{0}(x)= \begin{cases}b_{2}^{-2} & \text { for } x \in\left[0, x_{2}\right) \\ b_{1}^{-2} & \text { for } x \in\left[x_{2}, 1\right)\end{cases}
$$

and

$$
a(x)=\left\{\begin{array}{lc}
a_{0}(x-j) & \text { for } x \in[j, j+1), 0 \leq j \leq k-1 \\
b_{1}^{-2} & \text { elsewhere }
\end{array}\right.
$$

for $k \geq 2$, where $k$ is the number of identical cells. The period $l=1$. The normalized solutions $\psi_{1}(\lambda, x), \psi_{2}(\lambda, x)$,

$$
-\partial_{x} a(x) \partial_{x} \psi_{i}=\lambda^{2} \psi_{i}, \psi_{1}(\lambda, 0)=\left(b_{2}^{-2} \partial_{x} \psi_{2}\right)(\lambda, 0)=1, \psi_{2}(\lambda, 0)=\left(b_{2}^{-2} \partial_{x} \psi_{1}\right)(\lambda, 0)=0,
$$

satisfy (see [4])

$$
\begin{aligned}
& \psi_{1}(\lambda, x)=\left\{\begin{array}{cc}
\frac{1}{2} e^{i \lambda b_{2} x}+\frac{1}{2} e^{-i \lambda b_{2} x} & \text { for } x \in\left[0, x_{2}\right) \\
A e^{i \lambda b_{1} x}+B e^{-i \lambda b_{1} x} & \text { for } x \in\left[x_{2}, 1\right)
\end{array}\right. \\
& \psi_{2}(\lambda, x)=\left\{\begin{array}{cc}
-\frac{i b_{2}}{2 \lambda} e^{i \lambda b_{2} x}+\frac{i b_{2}}{2 \lambda} e^{-i \lambda b_{2} x} & \text { for } x \in\left[0, x_{2}\right) \\
C e^{i \lambda b_{1} x}+D e^{-i \lambda b_{1} x} & \text { for } x \in\left[x_{2}, 1\right)
\end{array}\right.
\end{aligned}
$$

with $A, B, C, D$ chosen such that $\psi_{j}$ and $a(x) \partial_{x} \psi_{j}$ are continuous at $x_{2}$ :

$$
\begin{aligned}
& A=\frac{1}{4 b_{2}}\left[\left(b_{2}+b_{1}\right) e^{i \lambda x_{2}\left(b_{2}-b_{1}\right)}+\left(b_{2}-b_{1}\right) e^{-i \lambda x_{2}\left(b_{2}+b_{1}\right)}\right], \\
& B=\frac{1}{4 b_{2}}\left[\left(b_{2}+b_{1}\right) e^{-i \lambda x_{2}\left(b_{2}-b_{1}\right)}+\left(b_{2}-b_{1}\right) e^{i \lambda x_{2}\left(b_{2}+b_{1}\right)}\right], \\
& C=\frac{i}{4 \lambda}\left[-\left(b_{2}+b_{1}\right) e^{i \lambda x_{2}\left(b_{2}-b_{1}\right)}+\left(b_{2}-b_{1}\right) e^{-i \lambda x_{2}\left(b_{2}+b_{1}\right)}\right], \\
& D=\frac{i}{4 \lambda}\left[\left(b_{2}+b_{1}\right) e^{-i \lambda x_{2}\left(b_{2}-b_{1}\right)}-\left(b_{2}-b_{1}\right) e^{i \lambda x_{2}\left(b_{2}+b_{1}\right)}\right] .
\end{aligned}
$$

We get the monodromy matrix

$$
M_{\lambda}=\left(\begin{array}{cc}
\alpha & \beta \\
\gamma & \delta
\end{array}\right)=\left(\begin{array}{cc}
\psi_{1}(\lambda, 1) & \lambda \psi_{2}(\lambda, 1) \\
\frac{1}{\lambda}\left(\frac{1}{b_{1}^{2}} \partial_{x} \psi_{1}\right)(\lambda, 1) & \left(\frac{1}{b_{1}^{2}} \partial_{x} \psi_{2}\right)(\lambda, 1)
\end{array}\right)
$$

with

$$
\begin{aligned}
& \alpha=\psi_{1}(\lambda, 1)=\frac{b_{2}+b_{1}}{2 b_{2}} \cos \lambda\left[b_{1}\left(1-x_{2}\right)+x_{2} b_{2}\right]+\frac{b_{2}-b_{1}}{2 b_{2}} \cos \lambda\left[b_{1}\left(1-x_{2}\right)-x_{2} b_{2}\right], \\
& \beta=\lambda \psi_{2}(\lambda, 1)=\frac{b_{2}+b_{1}}{2} \sin \lambda\left[b_{1}\left(1-x_{2}\right)+x_{2} b_{2}\right]-\frac{b_{2}-b_{1}}{2} \sin \lambda\left[b_{1}\left(1-x_{2}\right)-x_{2} b_{2}\right], \\
& \gamma=\frac{1}{\lambda}\left(\frac{1}{b_{1}^{2}} \partial_{x} \psi_{1}\right)(\lambda, 1)=-\frac{b_{2}+b_{1}}{2 b_{1} b_{2}} \sin \lambda\left[b_{1}\left(1-x_{2}\right)+x_{2} b_{2}\right]-\frac{b_{2}-b_{1}}{2 b_{1} b_{2}} \sin \lambda\left[b_{1}\left(1-x_{2}\right)-x_{2} b_{2}\right], \\
& \delta=\left(\frac{1}{b_{1}^{2}} \partial_{x} \psi_{2}\right)(\lambda, 1)=\frac{b_{2}+b_{1}}{2 b_{1}} \cos \lambda\left[b_{1}\left(1-x_{2}\right)+x_{2} b_{2}\right]-\frac{b_{2}-b_{1}}{2 b_{1}} \cos \lambda\left[b_{1}\left(1-x_{2}\right)-x_{2} b_{2}\right] .
\end{aligned}
$$


Then

$$
\operatorname{tr}\left(M_{\lambda}\right)=\alpha+\delta=(\rho+1) \cos \lambda\left[b_{1}\left(1-x_{2}\right)+x_{2} b_{2}\right]-(\rho-1) \cos \lambda\left[b_{1}\left(1-x_{2}\right)-x_{2} b_{2}\right],
$$

where $\rho=\frac{b_{2}^{2}+b_{1}^{2}}{2 b_{2} b_{1}}$. The Bloch quasi-momentum $\theta=\theta(\lambda)$ satisfy

$$
2 \cos \theta=\operatorname{tr} M_{\lambda}
$$

The first formula in (22) implies that the one cell medium is perfectly transparent: $\left|t_{1}\left(\lambda_{0}\right)\right|^{2}=$ $1,\left|r_{1}\left(\lambda_{0}\right)\right|^{2}=0$, if $\alpha-\delta=0, b_{1} \gamma+\frac{\beta}{b_{1}}=0$. We get $\lambda=\lambda_{0}=\pi m / x_{2} b_{2}$.

Note that there is a resonance $\lambda \in \operatorname{Res}\left(P_{1}\right)$ for the one cell operator such that $\operatorname{Re} \lambda=\lambda_{0}$ (see equation (35) with $b_{3}=b_{1}$ and $x_{1}=0$ ).

If $\lambda_{0}=\frac{\pi m}{x_{2} b_{2}}$ then we have

$$
2 F\left(\lambda_{0}\right)=2 \cos \theta\left(\lambda_{0}\right)= \pm 2 \cos \left(\frac{\left(1-x_{2}\right) b_{1}}{x_{2} b_{2}} \pi m\right), \text { if } m \text { is even (odd). }
$$

Thus in general situation, $\frac{\left(1-x_{2}\right) b_{1}}{x_{2} b_{2}} \notin \mathbb{Q}$, non-rational, $\lambda_{0}$ is an interior point of a spectral band.

If $x_{2} b_{2}=\left(1-x_{2}\right) b_{1}$ then the Lyapunov function satisfies

$$
2 F(\lambda)=(\rho+1) \cos \left(2 \lambda b_{2} x_{2}\right)-(\rho-1)
$$

and we have $F\left(\lambda_{0}\right)=1, F^{\prime}\left(\lambda_{0}\right)=0$ and $F^{\prime \prime}\left(\lambda_{0}\right) \neq 0$. Hence $\lambda_{0}$ is degenerate band edge.

The non-degenerate band edge is then given by the equation

$$
(\rho+1) \cos \left(2 \lambda b_{2} x_{2}\right)-(\rho-1)=-2 \Leftrightarrow \lambda=\frac{1}{2 b_{2} x_{2}}\left( \pm \arccos \left(\frac{\rho-3}{\rho+1}\right)+2 \pi n\right) .
$$

\section{Explicit construction of the Resolvent and RESOnAnCES}

In this section we define the resonances as the poles of the analytic continuation of the resolvent $R(\lambda)$ to $\mathbb{C}_{-}$.

4.1. Representation of the resolvent. In this section we revue some formulas used by Valeria Banica in [4, where she considered the local and global dispersion and the Stricharts inequalities for certain one-dimensional Schrödinger and wave equations with step-like coefficients. The systems are described by the one-dimensional Schrödinger equation

$$
\left\{\begin{aligned}
\left(i \partial_{t}+\partial_{x} a(x) \partial_{x}\right) u(t, x) & =0 \text { for }(t, x) \in(0, \infty) \times \mathbb{R} \\
u(0, x) & =u_{0}(x) \in L^{2}(\mathbb{R})
\end{aligned}\right.
$$

or by the one-dimensional wave equation

$$
\left\{\begin{aligned}
\left(\partial_{t}^{2}-\partial_{x} a(x) \partial_{x}\right) v(t, x) & =0 \text { for }(t, x) \in \mathbb{R} \times \mathbb{R} \\
v(0, x) & =u_{0}(x) \in L^{2}(\mathbb{R}), \\
\partial_{t} v(0, x) & =0
\end{aligned}\right.
$$

for a positive step-like function $a(x)$ with a finite number of discontinuities.

Consider a partition of the real axis

$$
-\infty=x_{0}<x_{1}<x_{2}<\ldots<x_{n-1}<x_{n}=\infty
$$

and a step function

$$
a(x)=b_{i}^{-2} \text { for } x \in\left(x_{i-1}, x_{i}\right)
$$

where $b_{i}$ are positive numbers.

The operator $P:=-\partial_{x} a(x) \partial_{x}$ defined from $\left\{u \in H^{1}(\mathbb{R}), a \partial_{x} u \in H^{1}(\mathbb{R})\right\}$ to $L^{2}(\mathbb{R})$ is selfadjoint. For $\operatorname{Im} \lambda>0$, we define $R(\lambda) v=\left(-\partial_{x} a(x) \partial_{x}-\lambda^{2}\right)^{-1} v$ the resolvent of $P$. 
Our choice of the spectral parameter $\lambda$ is related to $\omega$ in 4 by $\lambda=i \omega$. We use the expression for the resolvent obtained in [4].

On each interval $\left(x_{i}, x_{i+1}\right) R(\lambda) v$ is a finite sum of terms

$$
R(\lambda) v(x)=-\sum_{\text {finite }} C e^{i \lambda \beta(x)} \int_{I\left(x_{i}\right)} \frac{v(y)}{2 i \lambda} \frac{e^{ \pm i \lambda b_{i} y}}{\operatorname{det} D_{n}(-i \lambda)} d y-\int_{-\infty}^{\infty} \frac{v(y)}{2 i \lambda} b_{i} e^{i \lambda b_{i}|x-y|} d y
$$

where $I\left(x_{i}\right)$ is either $\left(-\infty, x_{i}\right)$ or $\left(x_{i}, \infty\right)$ and

$$
\operatorname{det} D_{2}(-i \lambda)=\left(b_{1}+b_{2}\right) e^{i \lambda x_{1}\left(b_{2}-b_{1}\right)} \text {. }
$$

In [4] Banica defines all det $D_{n}$ by induction. Let

$$
\operatorname{det} \widetilde{D_{2}(-i \lambda)}=\left(b_{2}-b_{1}\right) e^{-i \lambda x_{1}\left(b_{1}+b_{2}\right)} \text {. }
$$

We have the following induction relations for $n \geq 3$

$$
\begin{aligned}
& \operatorname{det} D_{n}=e^{i \lambda b_{n} x_{n-1}}\left[\left(b_{n-1}-b_{n}\right) e^{i \lambda b_{n-1} x_{n-1}} \operatorname{det} \widetilde{D_{n-1}}-\left(b_{n-1}+b_{n}\right) e^{-i \lambda b_{n-1} x_{n-1}} \operatorname{det} D_{n-1}\right] \\
& \operatorname{det} \widetilde{D_{n}}=e^{-i \lambda b_{n} x_{n-1}}\left[\left(b_{n-1}-b_{n}\right) e^{-i \lambda b_{n-1} x_{n-1}} \operatorname{det} D_{n-1}-\left(b_{n-1}+b_{n}\right) e^{i \lambda b_{n-1} x_{n-1}} \operatorname{det} \widehat{D_{n-1}}\right] .
\end{aligned}
$$

We define for $n \geq m \geq 2$

$$
Q_{m}(-i \lambda)=e^{2 i \lambda b_{m} x_{m}} \frac{\operatorname{det} \widetilde{D_{m}}}{\operatorname{det} D_{m}}, d_{m-1}=\frac{b_{m-1}-b_{m}}{b_{m-1}+b_{m}} .
$$

Then we have for $n \geq 3$

$$
\operatorname{det} D_{n}(i \lambda)=\left(b_{1}+b_{2}\right) e^{i \lambda\left(b_{2}-b_{1}\right) x_{1}} \Pi_{j=2 \ldots n-1}\left(b_{j}+b_{j+1}\right) e^{-i \lambda\left(b_{j}-b_{j+1}\right) x_{j}}\left(1-d_{j} Q_{j}(-i \lambda)\right) .
$$

We have induction formula on the $Q_{m}$ 's

$$
Q_{m}(-i \lambda)=e^{2 i \lambda b_{m}\left(x_{m}-x_{m-1}\right)} \frac{-d_{m-1}+Q_{m-1}(-i \lambda)}{1-d_{m-1} Q_{m-1}(-i \lambda)} .
$$

Note that a linear-fractional transform on the unit disc occurs in (34) for $\operatorname{Im} \lambda \geq 0$.

If $\operatorname{Im} \lambda \leq 0$ then $\left|e^{2 i \lambda b_{m}\left(x_{m}-x_{m-1}\right)}\right| \geq 1$. We use that $\left|d_{n}\right|<1$ and for any $n$ we can find $\epsilon_{n}>0$ such that for every complex $\lambda$ with

the estimate

$$
\operatorname{Im} \lambda \geq-\epsilon_{n}
$$

holds and gives by induction

$$
\left|Q_{2}(-i \lambda)\right|=\left|d_{1} e^{2 i \lambda b_{2}\left(x_{2}-x_{1}\right)}\right|<1
$$

$$
\left|Q_{m}(-i \lambda)\right|<1, n \geq m \geq 2 .
$$

Hence $\left(\operatorname{det} D_{n}(-i \lambda)\right)^{-1}$ is uniformly bounded and well defined in this region, which contains the real axis. Therefore $i \lambda R(\lambda) u_{0}(x)$ can be analytically continued. The spectral theorem gives

Lemma 2. The solution of the Schrödinger equation (30) verifies

$$
u(t, x)=\int_{-\infty}^{\infty} e^{i t \lambda^{2}} \lambda R(\lambda) u_{0}(x) \frac{d \lambda}{\pi} .
$$

The solution of the wave equation (31) verifies

$$
v(t, x)=\int_{-\infty}^{\infty} e^{i t \lambda} i \lambda R(\lambda) u_{0}(x) \frac{d \lambda}{2 \pi} .
$$


Due to formula (32), the resonance spectrum Res $(P)$ consist of zeros of $\operatorname{det} D_{n}(-i \lambda)$ or equivalently the zeros of $1-d_{n-1} Q_{n-1}(-i \lambda)$ as by (33), equation $\operatorname{det} D_{n}(-i \lambda)=0$ is equivalent to

$$
Q_{n-1}(-i \lambda)=\frac{1}{d_{n-1}}
$$

By considerations before Lemma 2, for each $n \geq 2$ there is $\epsilon_{n}>0$ such that all resonances verify $\operatorname{Im} \lambda<-\epsilon_{n}$. For $n=2$ there are no zeros.

For $n=3$ the resonances are solutions of the equation $\operatorname{det} D_{3}(-i \lambda)=0, \lambda=\lambda_{1}+i \lambda_{2}, \lambda_{j} \in \mathbb{R}$, with constant imaginary part:

$$
\lambda_{1}=\frac{\pi m}{2 b_{2}\left(x_{2}-x_{1}\right)}, \lambda_{2}=-\frac{1}{2 b_{2}\left(x_{1}-x_{2}\right)} \ln \left|\frac{\left(b_{2}-b_{3}\right)\left(b_{2}-b_{1}\right)}{\left(b_{2}+b_{3}\right)\left(b_{1}+b_{2}\right)}\right|<0,
$$

where $m=0, \pm 2, \pm 4 \ldots$, is even, if $\left(b_{2}-b_{3}\right)\left(b_{2}-b_{1}\right)>0$ or $m= \pm 1, \pm 3, \pm 5 \ldots$, is odd, if $\left(b_{2}-b_{3}\right)\left(b_{2}-b_{1}\right)<0$.

For $n>3$ the resonance spectrum can be obtained numerically using the induction relations (33).

4.2. Locally periodic media. Suppose that the profile of $a(x)$ consist of a finite number identical elements, obtained by juxtaposing of $k$ unit cells. Outside the interval $[0, k)$ the coefficient is constant. We make the following choice: suppose $n=2 k+1$ odd, $b_{1}=b_{3}=b_{5}=\ldots=b_{n}$, $b_{2}=b_{4}=\ldots=b_{n-1}$, and put

$$
x_{1}=0,0<x_{2}<1, x_{3}=1, d=\frac{b_{2}-b_{1}}{b_{2}+b_{1}}, \lambda=\lambda_{1}+i \lambda_{2}, \lambda_{1} \in \mathbb{R}, \lambda_{2}<0 .
$$

We have $x_{2 k+1}=k, x_{2 k}=k-1+x_{2}$. Let

$$
a_{0}(x)=\left\{\begin{array}{cc}
b_{2}^{-2} & \text { for } x \in\left[0, x_{2}\right) \\
b_{1}^{-2} & \text { for } x \in\left[x_{2}, 1\right)
\end{array}\right.
$$

and

$$
a(x)=\left\{\begin{array}{lc}
a_{0}(x-j) & \text { for } x \in[j, j+1), 0 \leq j \leq k-1 \\
b_{1}^{-2} & \text { elsewhere }
\end{array}\right.
$$

for $k \geq 2$. Here $k$ is the number of identical cells.

The function $a(x)$ is called locally periodic or finite periodic on the interval $[0, k)$ with $k$ cells.

Then, with $n=2 k+1$,

$$
\operatorname{det} D_{n}(-i \lambda)=\left(b_{1}+b_{2}\right)^{n-1} e^{-i \lambda\left(b_{2}-b_{1}\right) k x_{2}} \prod_{i=2, \ldots, n-1}\left(1-(-1)^{i} d Q_{i}(-i \lambda)\right),
$$

where

$$
Q_{2 k}(-i \lambda)=e^{\left.2 i \lambda b_{2}\left(k-1+x_{2}\right)\right)} \frac{\operatorname{det} \tilde{D}_{2 k}}{\operatorname{det} D_{2 k}}, Q_{2 k+1}(-i \lambda)=e^{2 i \lambda b_{1} k} \frac{\operatorname{det} \tilde{D}_{2 k+1}}{\operatorname{det} D_{2 k+1}}
$$

For $k=1,2,3, \ldots$ the resonances are the solutions of the equation

$$
\operatorname{det} D_{2 k+1}(-i \lambda)=0
$$

or equivalently

$$
Q_{2 k}(-i \lambda)=\frac{1}{d}
$$


where

$$
\begin{aligned}
& Q_{2 k}=e^{2 i \lambda b_{2} x_{2}} \frac{d+Q_{2 k-1}}{1+d Q_{2 k-1}}, \quad Q_{2 k-1}=e^{2 i \lambda b_{1}\left(1-x_{2}\right)} \frac{-d+Q_{2 k-2}}{1-d Q_{2 k-2}} \\
& Q_{2}=e^{2 i \lambda b_{2} x_{2}} d .
\end{aligned}
$$

Note that if condition (6) is satisfied: $b_{2} x_{2}=b_{1}\left(1-x_{2}\right)$, then $Q_{m}(-i \lambda)$ as function of $\operatorname{Re} \lambda$ is periodic with the period $\pi / b_{2} x_{2}$, which implies the same property for any solutions of equation (40). The resonance spectrum is then periodic as stated in the Introduction, Theorem 1 .

\section{The poles of analytic continuation of the Reflection coefficient $r_{k}$ to $\mathbb{C}_{-}$}

Let $r_{k}$ be reflection coefficient (24). In this section we express $r_{k}$ using the iteration formulas in section 4 and extend $r_{k}(\lambda)$ to $\lambda \in \mathbb{C}_{-}$. We show explicitly that the resonances defined in (39) or (40) are the poles of $r_{k}$,

$$
\frac{1}{r_{k}}=0 \Leftrightarrow \operatorname{det} D_{2 k+1}(-i \lambda)=0 \Leftrightarrow Q_{2 k}(-i \lambda)=\frac{1}{d},
$$

We consider the equation $P_{1} u=-\partial_{x} a(x) \partial_{x} u=\lambda^{2} u$ corresponding to the system with one unit-cell, $a(x)=a_{0}(x)$, for $x \in[0,1)$ and $a=1 / b_{1}^{2}$ outside $[0,1)$. Taking solution

$$
u(x)=A_{0} e^{i \lambda b_{1} x}+A_{0}^{\prime} e^{-i \lambda b_{1} x}, x<0 \text { and } u(x)=\tilde{A}_{0} e^{i \lambda b_{1} x}+\tilde{A}_{0}^{\prime} e^{-i \lambda b_{1} x}, x>1,
$$

the matching conditions imply

$$
\left(\begin{array}{c}
\tilde{A}_{0} \\
\tilde{A}_{0}^{\prime}
\end{array}\right)=T\left(\begin{array}{c}
A_{0} \\
A_{0}^{\prime}
\end{array}\right)
$$

where $T$ is called transmission matrix (see [1]).

Then coefficients of the solution of the problem with $k$-unit cells $P_{k} u=-\partial_{x} a(x) \partial_{x} u=\lambda^{2} u$ can be calculated by iteration. Taking solution

$$
u(x)=A_{-1} e^{i \lambda b_{1} x}+A_{-1}^{\prime} e^{-i \lambda b_{1} x}, x<0 \text { and } u(x)=\tilde{A}_{k} e^{i \lambda b_{1} x}+\tilde{A}_{k}^{\prime} e^{-i \lambda b_{1} x}, x>k,
$$

the coefficients of the two external regions $x<0$ and $x>k$ are related by

$$
\left(\begin{array}{c}
A_{k} \\
A_{k}^{\prime}
\end{array}\right)=\tilde{T}(\lambda)\left(\begin{array}{c}
A_{-1} \\
A_{-1}^{\prime}
\end{array}\right)
$$

where

$$
\tilde{T}(\lambda)=\left(\begin{array}{cc}
e^{-i l(k+1) \lambda b_{1}} & 0 \\
0 & e^{i l(k+1) \lambda b_{1}}
\end{array}\right) Q^{k}\left(\begin{array}{cc}
e^{i l \lambda b_{1}} & 0 \\
0 & e^{-i l \lambda b_{1}}
\end{array}\right)=
$$

where

$$
Q=\left(\begin{array}{ll}
Q_{11}(\lambda) & Q_{12}(\lambda) \\
Q_{21}(\lambda) & Q_{22}(\lambda)
\end{array}\right), \operatorname{det} Q=1, Q_{12}(\lambda)=Q_{21}(-\lambda), Q_{11}(-\lambda)=Q_{22}(\lambda)
$$

is called iteration matrix

$$
Q(\lambda)=D(\lambda) T(\lambda), D=\left(\begin{array}{cc}
e^{i \lambda b_{1}} & 0 \\
0 & e^{-i \lambda b_{1}}
\end{array}\right) .
$$

Next we relate the iteration matrix $Q$ and the monodromy matrix $M_{\lambda}$.

Relation between the Cauchy data and coefficients in (44) is given by

$$
\left(\begin{array}{c}
u(0) \\
\frac{\left(a \partial_{x} u\right)(\lambda, 0)}{\lambda}
\end{array}\right)=\left(\begin{array}{cc}
1 & 1 \\
\frac{i}{b_{1}} & -\frac{i}{b_{1}}
\end{array}\right)\left(\begin{array}{c}
A_{0} \\
A_{0}^{\prime}
\end{array}\right)=L\left(\begin{array}{c}
A_{0} \\
A_{0}^{\prime}
\end{array}\right)
$$


and

$$
\left(\begin{array}{c}
u(1) \\
\frac{\left(a \partial_{x} u\right)(\lambda, 1)}{\lambda}
\end{array}\right)=\left(\begin{array}{cc}
1 & 1 \\
\frac{i}{b_{1}} & -\frac{i}{b_{1}}
\end{array}\right)\left(\begin{array}{cc}
e^{i \lambda b_{1}} & 0 \\
0 & e^{-i \lambda b_{1}}
\end{array}\right)\left(\begin{array}{c}
\tilde{A}_{0} \\
\tilde{A}_{0}^{\prime}
\end{array}\right)=L D\left(\begin{array}{c}
\tilde{A}_{0} \\
\tilde{A}_{0}^{\prime}
\end{array}\right) .
$$

Using

$$
M_{\lambda}\left(\begin{array}{c}
u(0) \\
\frac{\left(a \partial_{x} u\right)(\lambda, 0)}{\lambda}
\end{array}\right)=\left(\begin{array}{c}
u(1) \\
\frac{\left(a \partial_{x} u\right)(\lambda, 1)}{\lambda}
\end{array}\right)
$$

and definition of $T$, equation (43), we get

$$
M_{\lambda}=L D T L^{-1}=L Q L^{-1}, \text { with } L=\left(\begin{array}{cc}
1 & 1 \\
\frac{i}{b_{1}} & -\frac{i}{b_{1}}
\end{array}\right) .
$$

We have

$$
\begin{aligned}
& Q_{12}(\lambda)=e^{i \lambda\left(1-x_{2}\right) b_{1}} \frac{b_{2}^{2}-b_{1}^{2}}{4 b_{1} b_{2}}\left(e^{-i \lambda b_{2} x_{2}}-e^{i \lambda b_{2} x_{2}}\right) \\
& Q_{22}(\lambda)=e^{-i \lambda b_{1}\left(1-x_{2}\right)}\left((r+1) e^{-i \lambda b_{2} x_{2}}-(r-1) e^{i \lambda b_{2} x_{2}}\right) .
\end{aligned}
$$

As

$$
\begin{aligned}
& \operatorname{det} \tilde{D}_{3}=e^{-i \lambda b_{1} x_{2}}\left(b_{2}^{2}-b_{1}^{2}\right)\left(e^{-i \lambda b_{2} x_{2}}-e^{i \lambda b_{2} x_{2}}\right), \\
& \operatorname{det} D_{3}=e^{i \lambda b_{1} x_{2}}\left(\left(b_{2}-b_{1}\right)^{2} e^{i \lambda b_{2} x_{2}}-\left(b_{2}+b_{1}\right)^{2} e^{-i \lambda b_{2} x_{2}}\right),
\end{aligned}
$$

then

$$
Q_{12}(\lambda)=\frac{e^{i \lambda b_{1}}}{4 b_{1} b_{2}} \operatorname{det} \tilde{D}_{3}, Q_{22}(\lambda)=-\frac{e^{-i \lambda b_{1}}}{4 b_{1} b_{2}} \operatorname{det} D_{3}
$$

Using the iteration relations between $\operatorname{det} \tilde{D}_{2 k-1}$, $\operatorname{det} D_{2 k-1}$ and $\operatorname{det} \tilde{D}_{2 k+1}$, det $D_{2 k+1}$ we get

Lemma 3. We have

$$
Q^{k}=\left(\begin{array}{ll}
Q_{22}^{(k)}(-\lambda) & Q_{12}^{(k)}(\lambda) \\
Q_{12}^{(k)}(-\lambda) & Q_{22}^{(k)}(\lambda)
\end{array}\right)
$$

where

$$
Q_{12}^{(k)}=\frac{e^{k i \lambda b_{1}}}{\left(4 b_{1} b_{2}\right)^{k}} \operatorname{det} \tilde{D}_{2 k+1}, Q_{22}^{(k)}=-\frac{e^{-i k \lambda b_{1}}}{\left(4 b_{1} b_{2}\right)^{k}} \operatorname{det} D_{2 k+1} .
$$

Using (48) we have also

$$
Q^{k}=L^{-1} M_{\lambda}^{k} L=\left(\begin{array}{cc}
\frac{1}{2}\left(\alpha_{k}+\beta_{k} \frac{i}{b_{1}}\right)-\frac{i b_{1}}{2}\left(\gamma_{k}+\delta_{k} \frac{i}{b_{1}}\right) & \frac{1}{2}\left(\alpha_{k}-\beta_{k} \frac{i}{b_{1}}\right)-\frac{i b_{1}}{2}\left(\gamma_{k}-\delta_{k} \frac{i}{b_{1}}\right) \\
\frac{1}{2}\left(\alpha_{k}+\beta_{k} \frac{i}{b_{1}}\right)+\frac{i b_{1}}{2}\left(\gamma_{k}+\delta_{k} \frac{i}{b_{1}}\right) & \frac{1}{2}\left(\alpha_{k}-\beta_{k} \frac{i}{b_{1}}\right)+\frac{i b_{1}}{2}\left(\gamma_{k}-\delta_{k} \frac{i}{b_{1}}\right)
\end{array}\right) .
$$

Using equation (24) we get

$$
r_{k}=-\frac{\left(\alpha_{k}-\delta_{k}\right)+i\left(b_{1} \gamma_{k}+\frac{\beta_{k}}{b_{1}}\right)}{\alpha_{k}+\delta_{k}+i\left(b_{1} \gamma_{k}-\frac{\beta_{k}}{b_{1}}\right)}=-\frac{2 Q_{21}^{(k)}(\lambda)}{2 Q_{22}^{(k)}(\lambda)}=-\frac{Q_{21}^{(k)}(\lambda)}{Q_{22}^{(k)}(\lambda)} .
$$

Let first $k=1$. Using equation (49) we get

$$
Q_{21}(\lambda)=-e^{-i 2 \lambda\left(1-x_{2}\right) b_{1}} Q_{12}=-\frac{e^{-i \lambda\left(1-x_{2}\right) b_{1}} e^{i \lambda b_{1} x_{2}}}{4 b_{1} b_{2}} \operatorname{det} \tilde{D}_{3}(\lambda)
$$


Hence

$$
\begin{aligned}
r_{1} & =-\frac{e^{-2 i \lambda b_{1}\left(1-x_{2}\right)} e^{i \lambda b_{1}\left(1-x_{2}\right)} e^{i \lambda b_{1} x_{2}} \operatorname{det} \tilde{D}_{3}}{e^{-i \lambda b_{1}} \operatorname{det} D_{3}}= \\
& =-e^{-2 i \lambda b_{1}\left(1-x_{2}\right)} e^{2 i \lambda b_{1} x_{2}} \frac{\operatorname{det} \tilde{D}_{3}}{\operatorname{det} D_{3}}=-e^{-2 i \lambda b_{1}\left(1-x_{2}\right)} Q_{3}(-i \lambda) .
\end{aligned}
$$

For $k>1$ we use

$$
Q^{k}=\frac{\sin k \theta l}{\sin \theta l} Q-\frac{\sin (k-1) \theta l}{\sin \theta l} I=\left(\begin{array}{cc}
\frac{\sin k \theta l}{\sin \theta l} Q_{11}-\frac{\sin (k-1) \theta l}{\sin \theta l} & \frac{\sin k \theta l}{\sin \theta l} Q_{12} \\
\frac{\sin k \theta l}{\sin \theta l} Q_{21} & \frac{\sin k \theta l}{\sin \theta l} Q_{22}-\frac{\sin (k-1) \theta l}{\sin \theta l}
\end{array}\right)
$$

and get

$$
Q_{21}^{(k)}=-e^{-i 2 \lambda\left(1-x_{2}\right) b_{1}} Q_{12}^{(k)}=-e^{-i 2 \lambda\left(1-x_{2}\right) b_{1}} \frac{e^{k i \lambda b_{1}}}{\left(4 b_{1} b_{2}\right)^{k}} \operatorname{det} \tilde{D}_{2 k+1} .
$$

Hence, by (24),

$$
r_{k}=-\frac{Q_{21}^{(k)}}{Q_{22}^{(k)}}=-\frac{e^{-i 2 \lambda\left(1-x_{2}\right) b_{1}} e^{k i \lambda b_{1}} \operatorname{det} \tilde{D}_{2 k+1}}{e^{-i k \lambda b_{1}} \operatorname{det} D_{2 k+1}}=-e^{-i 2 \lambda\left(1-x_{x}\right) b_{1}} e^{2 k i \lambda b_{1}} \frac{\operatorname{det} \tilde{D}_{2 k+1}}{\operatorname{det} D_{2 k+1}} .
$$

We have

$$
r_{k}=-e^{-2 i \lambda b_{1}\left(1-x_{2}\right)} Q_{2 k+1}(-i \lambda)=-\frac{-d+Q_{2 k}(-i \lambda)}{1-d Q_{2 k}(-i \lambda)} .
$$

Thus we have proved that the poles of $r_{k}$ on $\mathbb{C}_{-}$coincide with the solutions of (40):

Hence, we have

$$
1-d Q_{2 k}(-i \lambda)=0 \Leftrightarrow Q_{2 k}(-i \lambda)=\frac{1}{d}
$$

Proposition 1. The reflection coefficient $r_{k}(\lambda)$ continuous to $\mathbb{C}_{-}$with the poles at the resonance spectrum $\operatorname{Res}\left(P_{k}\right)$.

\section{Convergence of the Resonances to the ReAl axis as $k \rightarrow \infty$}

In this section we prove that the resonances spectrum $\operatorname{Res}\left(P_{k}\right)$ converges to the real axes as $k \rightarrow \infty$.

First we note that the function $\theta(\lambda)=\arccos ((\alpha(\lambda)+\delta(\lambda)) / 2)$ has analytic continuation onto the domain $\mathbb{C} \backslash \bigcup_{n=1}^{\infty} g_{n}$ by the formula $\theta(\bar{\lambda})=\overline{\theta(\lambda)}$ and $\operatorname{Im} \theta(\lambda)<0$ for $\operatorname{Im} \lambda<0$. For $\lambda \in \bigcup_{n=1}^{\infty} g_{n}$ we set $\theta(\lambda)=\theta(\lambda-i 0)$ and $\theta$ is pure imaginary there.

By Proposition 1 the function $r_{k}(\lambda)$ is analytic in $\lambda \in \mathbb{C}_{-} \backslash \operatorname{Res}\left(P_{k}\right)$, and the formula

$$
r_{k}(\lambda)=-\frac{(\alpha-\delta)+i\left(b_{1} \gamma+\frac{\beta}{b_{1}}\right)}{2 \sin l \theta(\lambda) \frac{\cos k l \theta(\lambda)}{\sin k l \theta(\lambda)}+i\left(b_{1} \gamma-\frac{\beta}{b_{1}}\right)}
$$

extends to $\mathbb{C}_{-} \backslash \operatorname{Res}\left(P_{k}\right)$, where we have $\operatorname{Im} \theta(\lambda)<0$.

Furthermore, if $\operatorname{Im} \theta(\lambda)<0$, then

$$
\frac{\cos k \theta(\lambda)}{\sin k \theta(\lambda)} \rightarrow i \text { as } k \rightarrow \infty,
$$

and we have

$$
r_{k} \rightarrow \frac{i(\alpha-\delta)-\left(b_{1} \gamma+\frac{\beta}{b_{1}}\right)}{2 \sin l \theta(\lambda)+\left(b_{1} \gamma-\frac{\beta}{b_{1}}\right)}=\tilde{r}(\lambda) .
$$

Note that for $\lambda \in \mathbb{R}, \tilde{r}(\lambda)=\overline{r(-\lambda)}$. 
The limit extends also to $\lambda \in \cup_{n=1}^{\infty} g_{n}$, where $\theta(\lambda)$ is pure imaginary.

Let $g_{k}(\lambda), g(\lambda)$ denote the denominators of $r_{k}$ respectively $\tilde{r}$ for $\lambda \in \mathbb{C}_{-}$. Then

$$
g_{k}(\lambda) \rightarrow g(\lambda)=2 \sin l \theta(\lambda)+\left(b_{1} \gamma-\frac{\beta}{b_{1}}\right)
$$

uniformly on any compact subsets of $\mathbb{C}_{-}$and the limiting function $g$ is analytic on $\mathbb{C}_{-}$.

For any $k$ all zeros of $g_{k}$ have negative imaginary part: $\operatorname{Res}\left(P_{k}\right) \in \mathbb{C}_{-}$. Then, by the Hurwitz's theorem, the zeros of $g_{k}$ can only converge to the real axis.

\section{The LIMIt $\lim _{k \rightarrow \infty} r_{k}$ AS A FIXED POINT OF A SEQUENCE OF LINEAR-FRACTIONAL AUTOMORPHISMS OF THE UNIT DISK}

Equation (50) together with iteration relations (50) shows that for $\lambda \in \mathbb{R}$ real and for all $k=1,2,3, \ldots$ the reflection coefficients for the $k+1$ and $k$ cells media are related by $r_{k+1}=f_{\lambda}\left(r_{k}\right)$, where $f_{\lambda}$ is linear-fraction automorphism of the unit disk. Hence we get

$$
r_{k}=f_{\lambda}^{[k]}\left(r_{1}\right):=\underbrace{f_{\lambda} \circ f_{\lambda} \ldots f_{\lambda}}_{k \text { iterates }}\left(r_{1}\right) \text {. }
$$

Here $r_{1}$ is the reflection coefficient for $P_{1}$,

$$
r_{1}=-\frac{\left(1-e^{2 i \lambda b_{2} x_{2}}\right) d}{1-d^{2} e^{2 i \lambda b_{2} x_{2}}}
$$

and for $\lambda \in \mathbb{R}$ we have

$$
\left|r_{1}\right|^{2} \leq \frac{4 d^{2}}{1+4 d^{2}+d^{4}}<1
$$

In this section we will consider the limit of the sequence $f_{\lambda}^{[k]}$ as $k \rightarrow \infty$.

First we recall some well-known facts on the convergence behavior of a sequence $\left\{f^{[k]}\right\}$ when $f$ is general linear-fractional automorphism $f$ of the unit disc $D=\{z \in \mathbb{C} ;|z|<1\}$,

$$
f(z)=\frac{b-z}{1-\bar{b} z}, b \in D, z \in \mathbb{C} \backslash\{1 / \bar{b}\} .
$$

We refer to the paper of Burckel [5]) for the details.

In general situation $(b \neq 0), f$ has two fixed points $z_{1}, z_{2}$. There are three cases to consider.

Hyperbolic: $f$ has two (distinct) fixed points on $\partial D$

$$
\left|z_{1}\right|=\left|z_{2}\right|=1, z_{1} \neq z_{2}
$$

In this case the sequence $\left\{f^{[k]}\right\}$ converges uniformly on compact subsets in $D$ to one of these points.

Parabolic: $f$ has one (double) fixed point on $\partial D$

$$
z_{1}=z_{2}, \text { with }\left|z_{1}\right|=1 .
$$

In this case the sequence $\left\{f^{[k]}\right\}$ converges uniformly on compact subsets in $D$ to this fixed point.

Elliptic: $f$ has two fixed points: one fixed point $z_{1} \in D$ and one fixed point $z_{2}=1 / \bar{z} \notin D$. In this case either $f$ is periodic in the sense that $f^{[n]}=I$ for some $n$, or the orbit $\left\{f^{[k]} ; n \in \mathbb{N}\right\}$ is dense in the compact group of all conformal automorphisms of $D$ which fix $z_{1}$. 
We apply these results to $f=f_{\lambda}$. In order to simplify the formulas we suppose

$$
b_{2} x_{2}=b_{1}\left(1-x_{2}\right) .
$$

Then we have the following expression for $f_{\lambda}$ :

$$
f_{\lambda}(z)=\frac{-d+\eta \frac{d+\eta z}{1+d \eta z}}{1-d \eta \frac{d+\eta z}{1+d \eta z}}, \text { where } \eta:=e^{2 i \lambda b_{2} x_{2}} .
$$

In general situation $\eta \neq 1$, equation $f_{\lambda}(z)=z$ have solutions

$$
z_{1,2}=\frac{-(1+\eta) \pm \sqrt{(1+\eta)^{2}-4 d^{2} \eta}}{2 d \eta}=\frac{-\cos \left(\lambda b_{2} x_{2}\right) \pm \sqrt{\cos ^{2}\left(\lambda b_{2} x_{2}\right)-d^{2}}}{d e^{i \lambda b_{2} x_{2}}} .
$$

Note that

$$
\begin{aligned}
& \text { if } d^{2}<\cos ^{2}\left(\lambda b_{2} x_{2}\right)<1 \text { then }\left|z_{1}\right|<1,\left|z_{2}\right|>1 \text { and } z_{1} \cdot \overline{z_{2}}=1 \\
& \text { if } \cos ^{2}\left(\lambda b_{2} x_{2}\right) \leq d^{2} \text { then }\left|z_{1}\right|=\left|z_{2}\right|=1,
\end{aligned}
$$

and $z_{1}=z_{2}$ if $\cos ^{2}\left(\lambda b_{2} x_{2}\right)=d$.

Note that if (52) is satisfied then the Lyapunov function is given by (29), $F(\lambda)=(\rho+$ 1) $\cos ^{2}\left(\lambda b_{2} x_{2}\right)-\rho$. The inner points of the allowed bands $\bigcup_{n=1}^{\infty} b_{n}$ satisfy $|F(\lambda)|^{2}<1$ which is equivalent to $d^{2}<\cos ^{2}\left(\lambda b_{2} x_{2}\right)<1$. On the spectral gaps $\bigcup_{n=1}^{\infty} g_{n}$ we have $\cos ^{2}\left(\lambda b_{2} x_{2}\right)<d^{2}$. The non-generated edge point of a band is given by $\cos ^{2}\left(\lambda b_{2} x_{2}\right)=d^{2}$. The degenerated edge point $\lambda_{0}$ satisfy $\cos ^{2}\left(\lambda b_{2} x_{2}\right)=1 \Leftrightarrow \lambda_{0}=\pi m / b_{2} x_{2}$.

Hence we get the following version of the forth result in Theorem 2 ,

Proposition 2. If $\lambda \in \bigcup_{n=1}^{\infty} g_{n}$ then the linear fractional automorphism $f_{\lambda}$ has two fixed points $z_{1}, z_{2}$ of hyperbolic type: $\left|z_{1}\right|=\left|z_{2}\right|=1$. The sequence $r_{k}$ converges to $r$ as $k \rightarrow \infty$, where

$$
r=\lim _{k \rightarrow \infty} f_{\lambda}^{[k]}\left(r_{1}\right)
$$

is either $z_{1}$ or $z_{2}$.

If $\lambda$ is a non-degenerated band edge point: $F(\lambda)= \pm 1$ and $F^{\prime}(\lambda) \neq 0$, then $f_{\lambda}$ has one (double) fixed point $z_{1}=z_{2}$ and $\left|z_{1}\right|=1$.

We have

$$
r=z_{1}=\lim _{k \rightarrow \infty} r_{k}=\lim _{k \rightarrow \infty} f_{\lambda}^{[k]}\left(r_{1}\right) .
$$

If $\lambda=\lambda_{0}$ is degenerate band edge point $F\left(\lambda_{0}\right)= \pm 1, F^{\prime}\left(\lambda_{0}\right)=0$ (degenerate band edge) then $r_{k}(\lambda)=0$ for all $k=1,2,3 \ldots$

The Proposition is still valid if condition (52) is not imposed.

\section{Appendix A. Numerical calculations}

In this section we present some examples of the resonance spectrum. The resonances are solutions of the equations $D_{2 k+1}(-i \lambda)=0$, where $D_{n}, n=2 k+1$, are defined iteratively by (33), Section 4. The zeros of $D_{2 k+1}(-i \lambda)$ are calculated numerically by using the Newton procedure. Using Matlab we plot the resonance spectrum for the number of identical cells $k=3,4,5$. In the same figure we show the band spectrum for the corresponding periodic problem satisfying (4). The small circles on the real axis marks the position of $\lambda_{0}=\pi m /\left(x_{2} b_{2}\right)$ when the one-cell system is perfectly transparent: $\left|t_{1}\left(\lambda_{0}\right)\right|^{2}=1, \mid r_{1}\left(\left.\lambda_{0}\right|^{2}=0\right.$.

On Figure (11) condition (6) is satisfied: $b_{1}=1, b_{2}=4, x_{2}=0.2$.

On Figure (21) condition (6) is not satisfied: $b_{1}=1, b_{2}=3.8, x_{2}=0.2$.

On Figure (3) condition (6) is not satisfied: $b_{1}=3.8, b_{2}=1, x_{2}=0.8$. 
RESONANCE SPECTRUM FOR ONE-DIMENSIONAL LAYERED MEDIA
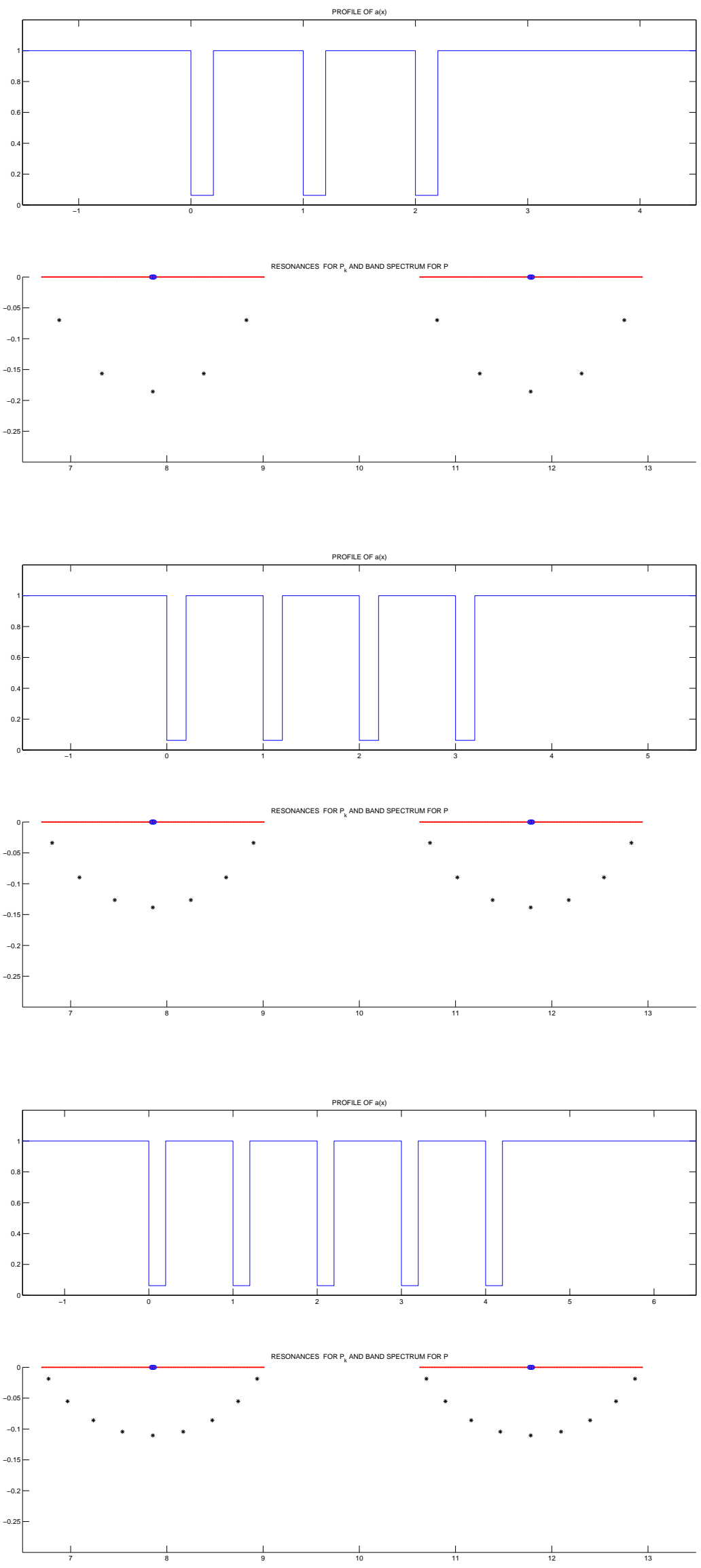

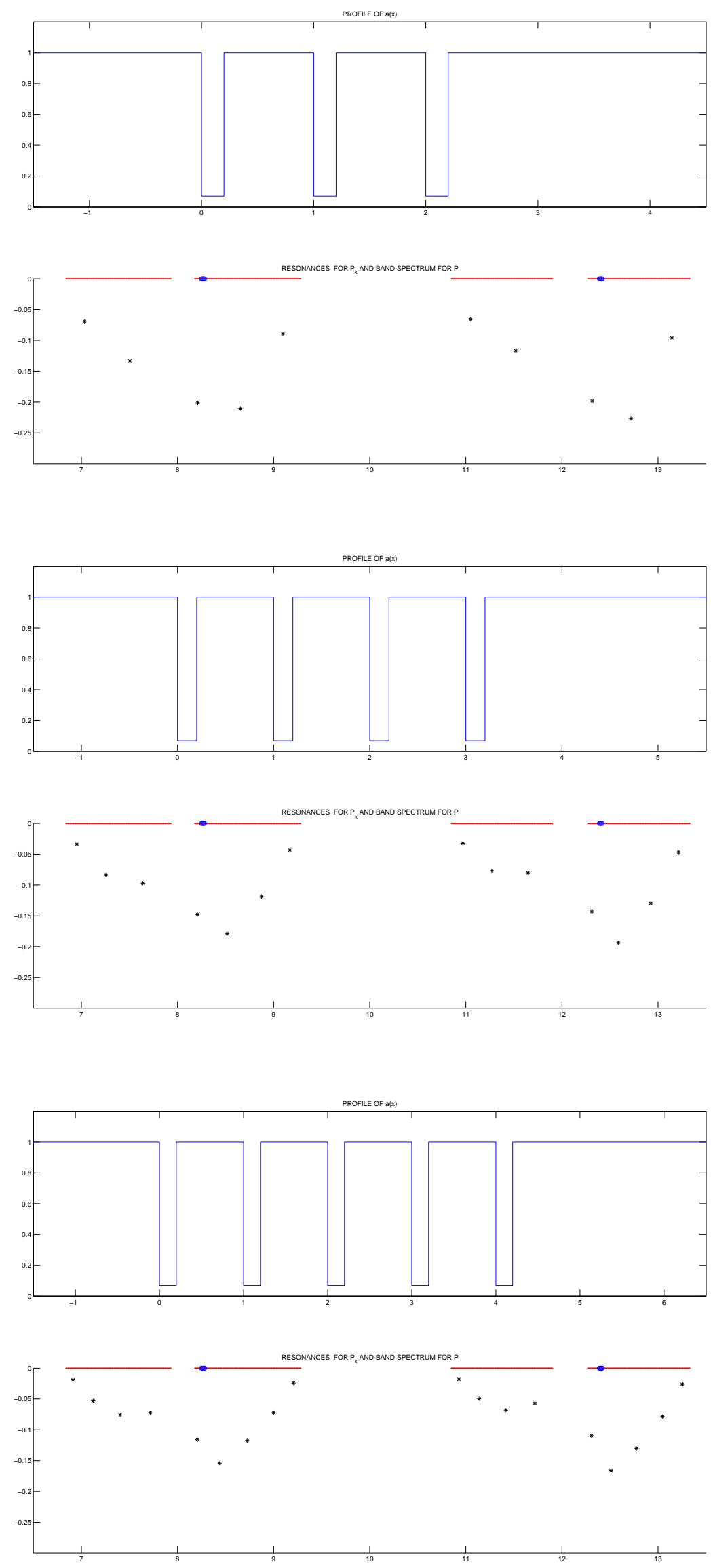
RESONANCE SPECTRUM FOR ONE-DIMENSIONAL LAYERED MEDIA
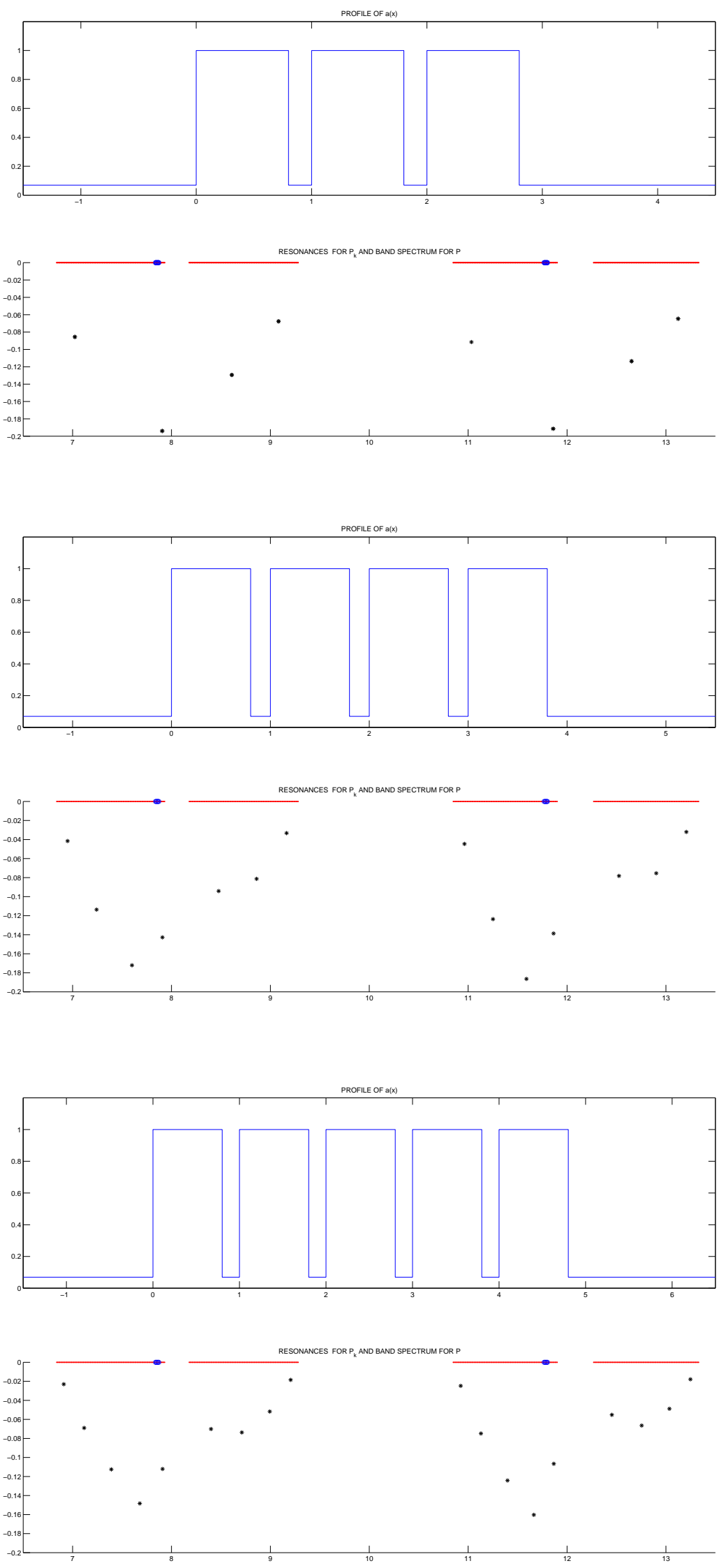


\section{REFERENCES}

[1] C. Cohen-Tannoudji and B. Diu and F. Laloë. Quantum Mechanics. New York: Wiley, 1977.

[2] B.M. Levitan and I.S. Sargsyan. Sturm-Lioville and Dirac Operators. Moscow, 1988 (in russian).

[3] F. Barra and P. Gaspard. Scattering in periodic systems: from resonances to band structure. J. Phys. A: Math. Gen., 32:3357-3375, 1999.

[4] Valeria Banica. Dispersion and Strichartz inequalities for Schrödinger equations with singular coefficients. SIAM J. Math. Anal., 35(4):868-883, 2003.

[5] R. B. Burckel. Iterating analytic self-maps of discs. The American Mathematical Monthly, 88(6):396-407, 1981.

[6] David J. Griffiths and Carl A. Steinke. Waves in locally periodic media. Am. J. Phys., 69(2):137-154, 2001.

[7] B. Vainberg S. Molchanov. Slowing down and reflection of waves in truncated periodic media. J.Func.An., 231:287-311, 2006.

Malmö University, School of Technology and Society, SE-205 06 Malmö, Sweden

E-mail address: ai@ts.mah.se 